\title{
ToxVec: Deep Language Model-Based Representation Learning for Venom Peptide Classification
}

${ }_{4}$ Meisam Ahmadi ${ }^{1}$, Mohammad Reza Jahed-Motlagh ${ }^{1, *}$, Ehsaneddin Asgari ${ }^{2}$, Adel Torkaman Rahmani ${ }^{1}$, and Alice C. McHardy ${ }^{2, *}$

6 'Department of Computer Engineering, Iran University of Science and Technology, Tehran, Iran

- ${ }^{2}$ Computational Biology of Infection Research, Helmholtz Center for Infection Research, 38124 Braunschweig, Germany

Corresponding author:

Mohammad Reza Jahed-Motlagh and Alice McHardy ${ }^{1, *}$

Email address: jahedmr@iust.ac.ir AND Alice.McHardy@helmholtz-hzi.de

\section{ABSTRACT}

\begin{abstract}
Venom is a mixture of substances produced by a venomous organism aiming at preying, defending, or intraspecific competing resulting in certain unwanted conditions for the target organism. Venom sequences are a highly divergent class of proteins making their machine learning-based and homology-based identification challenging. Prominent applications in drug discovery and healthcare, while having scarcity of annotations in the protein databases, made automatic identification of venom an important protein informatics task. Most of the existing machine learning approaches rely on engineered features, where the predictive model is trained on top of those manually designed features. Recently, transfer learning and representation learning resulted in significant advancements in many machine learning problem settings by automatically learning the essential features. This paper proposes an approach, called ToxVec, for automatic representation learning of protein sequences for the task of venom identification. We show that pre-trained language model-based representation outperforms the existing approaches in terms of the $\mathrm{F} 1 \mathrm{score}$ of both positive and negative classes achieving a macro-F1 of 0.89 . We also show that an ensemble classifier trained over multiple training sets constructed from multiple down-samplings of the negative class instances can substantially improve a macro-F1 score to 0.93 , which is 7 percent higher than the state-of-the-art performance.
\end{abstract}

Availability: The ToxVec application is available to use at https://github.com/meahmadi/ToxVec

\section{INTRODUCTION}

Venom is a mixture of enzymatic or non-enzymatic substances produced by the body of a venomous organism aiming at preying, defending, or intraspecific competing (Casewell et al., 2013) resulting in immobilizing or paralyzing the target organism. Venom has evolved independently multiple times throughout the tree of life, making the evolutionary study of venom a significant interest (Jenner et al., 2019). Being rich in having ion channels, G-protein-coupled receptors, and transporters have made venom an excellent source for therapeutics and drug discoveries (Lewis and Garcia, 2003; Prashanth et al., 2017). Despite prominent applications of venom in drug discovery and healthcare, only a small portion of proteins are annotated in large protein databases (UniProt/SwissProt) to be venom (Jungo et al., 2012) (Currently, 6,736 out of 563,082 protein sequences in Swiss-Prot). This gap motivates computational methods that can automatically and accurately identify venom peptides in the large protein datasets. The prediction of venoms versus non-venom sequences is not a trivial task protein classification task, where the use of BLAST-based approaches is challenging: venoms are often (i) evolved from non-toxic proteins 
(Hargreaves et al., 2014), (ii) and then have highly diverged (Linial et al., 2017). Several studies have proposed computational and machine learning-based methods for predicting or analyzing toxin/venom peptides (Cole and Brewer, 2019; Dao et al., 2017; Gacesa et al., 2016; Naamati et al., 2009; Ojeda et al., 2018; Pan et al., 2020; Wong et al., 2013). In the following, we summarize some of the recent machine learning supervised methods proposed for venom identification with available software/working servers which we could compare with our proposed ToxVec.

ClanTox (Naamati et al., 2009) is a machine learning-based classification of venom available as a web-server. In the ClanTox, each sequence is encoded into a vector of 545 global sequence features and the predictive model consisting of 10 boosted-stump classifiers is trained over the dataset of known venoms (Iba and Langley, 1992) scoring venoms on a scale of -1 to 1 . ToxClassifier (Gacesa et al., 2016) is an ensemble predictor using nine Support Vector Machine (SVM) (Cortes and Vapnik, 1995), Gradient Boosted Machine (GBM)(Friedman, 2002) and Generalised Linear Model (GLM) (Nelder and Wedderburn, 1972) classifiers over different combinations of features including sequence length, frequency of amino acids, amino acid dimer frequency, Hidden Markov Models (HMM) of tox-bit motifs (Starcevic et al., 2015), homology-based features (against a positive venom database).Toxify (Cole and Brewer, 2019) is a deep learning-based venom predictor employing Recurrent Neural Networks (RNN) and, in particular, the Gated Recurrent Units (GRUs) variation of RNN (Cho et al., 2014) for sequence modeling and ultimately prediction. For sequence encoding, toxify uses five Atchley factors per amino acid in the protein (Atchley et al., 2005). Similarly, in this paper, we propose a deep-learning approach for supervised training of the venom predictor model. However, instead of using manually extracted features, we propose a transfer learning framework. Similar to ProtVec (Asgari and Mofrad, 2015) and ProtVecX (Asgari, 2019; Asgari et al., 2019a), we use a skip-gram network (Bojanowski et al., 2017; Mikolov et al., 2013) which is analogous to language modeling. Subsequently, the pretrained network is fine-tuned for the venom classification task.

Recently, transfer learning resulted in significant advancements in many machine learning problem settings, particularly for inadequately annotated data (Bengio, 2012; Tan et al., 2018; Wolf et al., 2019). Transfer learning in machine learning refers to the use of the solution in a problem setting (source problem) with enough training samples/prior knowledge to solve a different problem (target problem) with less training samples/prior knowledge. Using a neural network trained relevant representations for a specific task for another task is also an instance of transfer learning through representation learning (Bengio, 2012; Tan et al., 2018). Combinations of being self-supervised and being general enough make neural language modeling an ideal candidate for transfer learning on the sequential data (Howard and Ruder, 2018). Afterward, the trained language modeling network can be fine-tuned for any particular task, even when only a limited number of annotations are available. Here we describe the use of Skip-gram (Bojanowski et al., 2017; Mikolov et al., 2013), one of the most successful architecture to perform transfer learning on natural language text for the task of venom prediction.

This paper shows that fine-tuning of language model-based representation outperforms the state-ofthe-art approaches in venom peptide classification. In addition, ensemble classifiers trained on resamples of negative samples (the major class) further improve the macro-F1 of both negative and positive classes.

\section{METHODS}

\subsection{Datasets}

For the ease of benchmarking, we use the dataset created and proposed by Toxify (Cole and Brewer, 2019) containing training and test protein sequences:

The Toxify training dataset contains (i) Positive examples: 6,133 venom protein sequences extracted from Swiss-Prot sequences annotated with annotation:(type: tissue specificity venom)), (i) Negative examples: 50,000 random protein sequences from Swiss-Prot satisfying the query NOT annotation:(type: tissue specificity venom), these sequences only include the sequences uploaded prior to June 2016 on Swiss-Prot.

The Toxify test dataset contains 274 verified venom protein sequences (2016-2018, not included in the training) and 94 verified non-venom protein sequences from the same time interval of (2016-2018). 


\subsection{Skip-gram analogous to Language Modeling}

Language modeling aims to assign a probability $P\left(w_{1}, w_{2}, \ldots, w_{N}\right)$ to a given sequence of elements (words, phrases, or amino acids in proteins) $w_{1}, w_{2}, \ldots, w_{N}$. Language modeling is a vital component in many language processing applications, particularly the applications containing language generation or the evaluation of text correctness, e.g., chat-bot or machine translation. Language modeling probability can be written as follows using the chain rule:

$$
P\left(w_{1}, w_{2}, \ldots, w_{N}\right)=P\left(w_{1}\right) \times P\left(w_{2} \mid w_{1}\right) \times P\left(w_{3} \mid w_{1}, w_{2}\right) \times \ldots \times P\left(w_{N} \mid w_{1}, \ldots, w_{N-1}\right)
$$

Requiring only raw data and being general enough has made language modeling a favorable task for transfer learning. Recently, transfer learning from the language modeling became a very popular method in natural language processing and bioinformatics and obtained state-of-the-art performance in many tasks (Asgari and Mofrad, 2015; Bengio, 2012; Howard and Ruder, 2018; Rao et al., 2019; Tan et al., 2018). A variety of language models are proposed in the literature. In this paper, we focus on Skip-gram neural network (depicted in Figure 1.1) whose objective is analogous to the objective of the language modeling task. However, in skip-gram the input and output are swapped and it predicts the surroundings (context) for a given textual unit. The objective of skip-gram is to maximize the following log-likelihood:

$$
\sum_{t=1}^{M} \sum_{c \in[t-N, t+N]} \log p\left(w_{c} \mid w_{t}\right)
$$

where $N$ is the surrounding window size around word $w_{t}, c$ is the context indices around index $t$, and $M$ is the corpus size in terms of the number of available words and context pairs. This probability of observing a context word $w_{c}$ given $w_{t}$ is parameterized using word embedding:

$$
p\left(w_{c} \mid w_{t} ; \theta\right)=\frac{e^{v_{c} \cdot v_{t}}}{\sum_{c^{\prime} \in \mathscr{C}} e^{v^{\prime} \cdot v_{t}}},
$$

where $\mathscr{C}$ denotes all existing contexts in the training data. However, iterating over all existing contexts is computationally expensive. This issue can be efficiently addressed by using negative sampling. In a negative sampling framework, we can rewrite Equation 1 as follows:

$$
\sum_{t=1}^{T}\left[\sum_{c \in[t-N, t+N]} \log \left(1+e^{-s\left(w_{t}, w_{c}\right)}\right)+\sum_{w_{r} \in \mathscr{N}_{t, c}} \log \left(1+e^{s\left(w_{t}, w_{r}\right)}\right)\right],
$$

where $\mathscr{N}_{t, c}$ denotes a set of randomly selected negative examples sampled from the vocabulary collection as non-contexts of $w_{t}$ and $s\left(w_{t}, w_{c}\right)=v_{t}^{\top} \cdot v_{c}$ (parameterization with the word vector $v_{t}$ and the context vector $v_{c}$ ) (Goldberg and Levy, 2014). The use of Skip-gram for protein sequences and transfer learning in protein informatics has been proposed by a number of recent works (Asgari et al., 2019a; Asgari and Mofrad, 2015; Wan and Zeng, 2016).

\subsection{Overview of Approach}

Here we describe our approach ToxVec in the use of language-model based representation for the classification of venom peptides. The ToxVec computational workflow has the following steps (as depicted in Figure 1):

1. Unsupervised Training of the Language Model-based Embeddings: In this step (Figure 1.1), we train a protein k-mer representation proposed in (Asgari and Mofrad, 2015), ProtVec. For this study, we used a recent version of ProtVec where the training is expanded from Swiss-Prot (containing $\approx 500 \mathrm{~K}$ sequences) to a much larger set, UniRef 90 , containing $\approx 115 \mathrm{M}$ protein sequences. Next, the protein sequences are divided into non-overlapping 3-mers by adding two starting symbols of \#\# and two ending symbols of @@. As detailed in (Asgari and Mofrad, 2015) and also shown in Figure 1, all three ways of splitting (based on the starting position for splitting) is done (i) to increase the training size to 


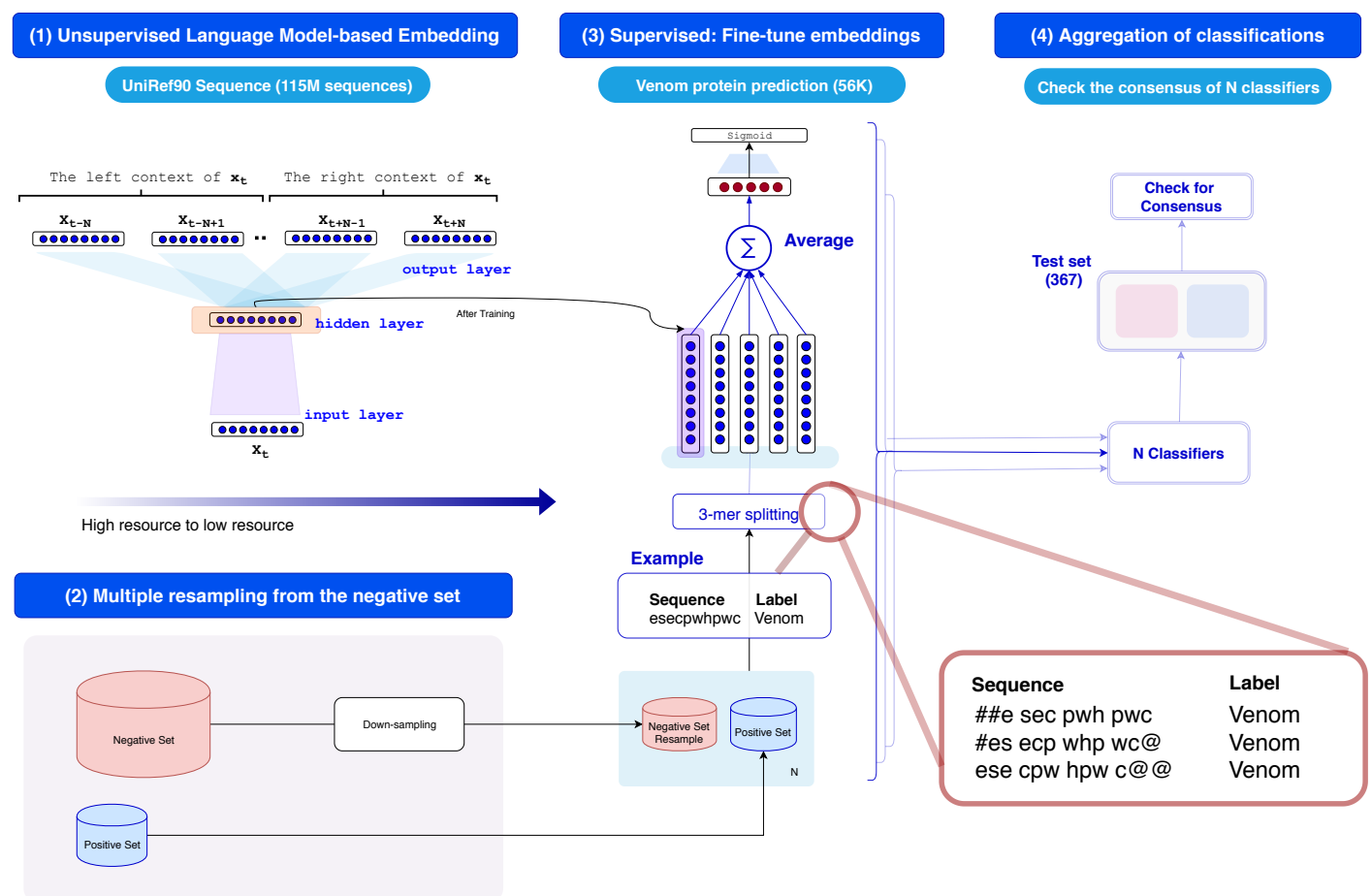

$\approx 115 M \times 3=445 M$ sequences of $\mathrm{k}$-mers and (ii) to capture all possible neighborhoods. The skip-gram network is trained on the mentioned collection of divided sequences, with the window size of 20 , and the vector size of 3000 .

Figure 1. Overview of the Tox Vec approach for the detection of venom proteins using fine-tuning of language model-based representations. The steps are detailed in the §1.3. (1) The first step is the training of Skip-gram embedding for protein k-mers over UniRef90, (2) We draw multiple $(\mathrm{N}=10)$ resamples from the major class (negative set), (3) We fine-tune the Skip-gram embeddings for the venom classification in the classification network, (4) The eventual output is the aggregated result from ( $N=10$ classifiers).

2. Multiple Resampling from the Major Class (negative) Since in the training dataset provided by (Cole and Brewer, 2019), the negative set is almost eight times larger than the positive set, the classifier is subject to be biased towards the negative class. To address this issue, we downsample the negative set to the positive set's size to mitigate this bias. In addition, next, to ensure the use of more negative samples, we perform $\mathrm{N}$ resamplings of the negative set and subsequently train $\mathrm{N}$ classifiers $(\mathrm{N}=10)$.

3. Supervised Fine-tuning of Embeddings for the Venom Classification For each resampled training set (in step 2), we train a classification network in the next step. As classification model, we used the fasttext model (Bojanowski et al., 2017), a simple but effective model for sentence classification in NLP: the input embeddings (here k-mer embedding) are averaged followed by a feedforward layer before the ending sigmoid layer produces the class conditional probabilities. For the k-mer embedding of the input sequences, we use the ProtVec embeddings detailed in the $1^{\text {st }}$ step. We fine-tune the $\mathrm{k}$ mers embedding in the course of supervised training. To investigate the role of pretrained ProtVec in classification performance, we repeat the same experiment with randomly initialized k-mer embedding.

Furthermore, since in the creation of embedding training corpus (step 1), each protein sequence is divided into three sequences of $\mathrm{k}$-mers $(\mathrm{k}=3)$, the test set sequences would also undergo the same procedure. Thus, at the inference time, for each test sequence, we would have three possible segmentations (e.g., esecpwhpwc $\rightarrow$ (1) \#\#e, sec, pwh, pwc (2) \#es, ecp, whp, wc@ (3) ese, cpw, hpw, c@@) and subsequently we would have three classification outcomes. This way, we have three binary outcomes for 

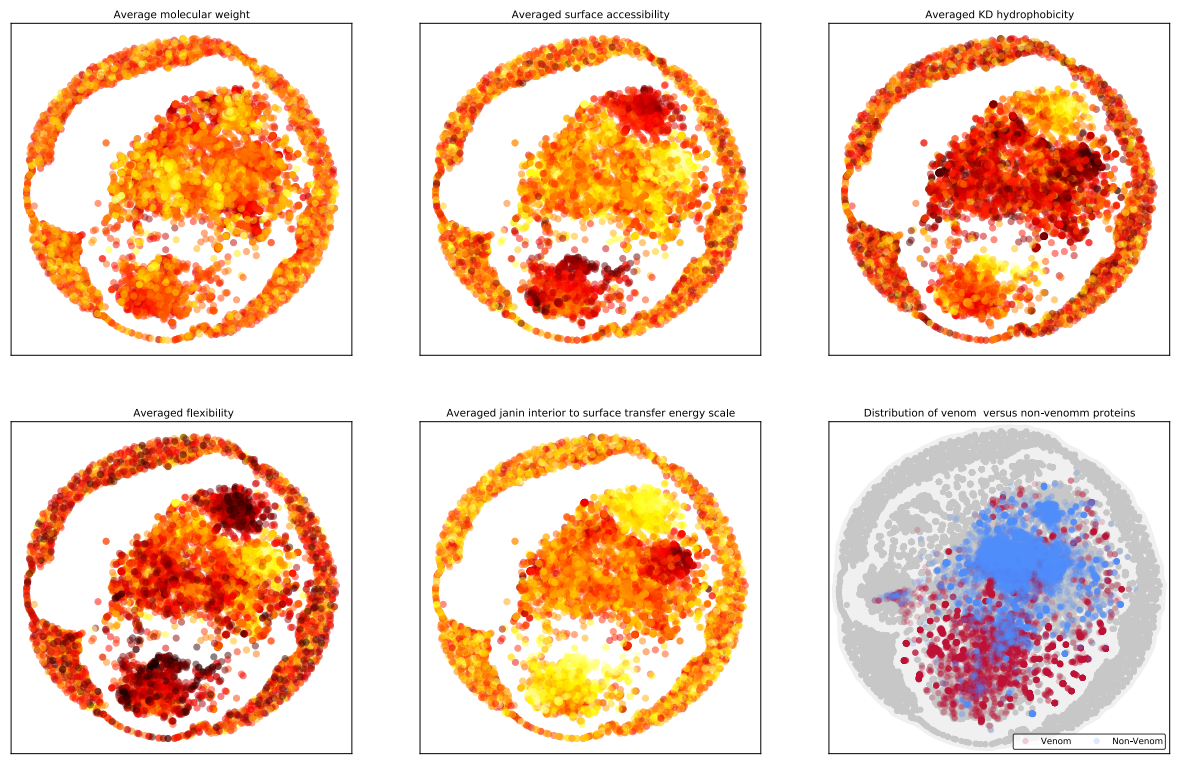

Figure 2. Distribution of biophysical and biochemical properties in the protein trimers (except (f)) and in venom sequences versus non-venoms (f) in the embedding space visualized using t-SNE. The five heatmaps scatter plots of biophysical properties (Figures (a) to (e)) show the standardized scales averaged for each trimer. Figure (f) shows the distribution of training instances of venom (colored in red) versus non-venom (colored in blue) in this space.
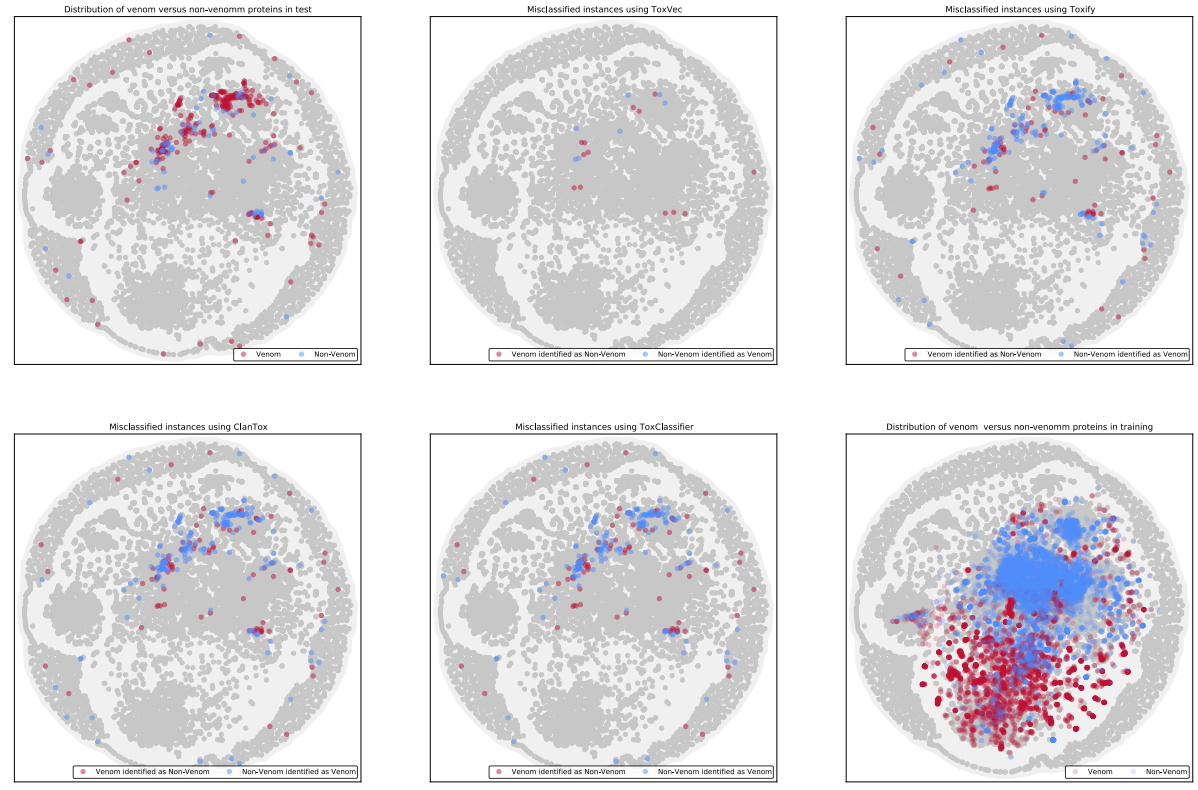

Figure 3. Visualization of test (a), train (f), and misclassified instances ((b) to (e)) using different existing venom predictive models in the embedding space of protein k-mers (timers) for venom prediction. The ToxVec (b), Toxify (c), ClanTox (d), ToxClassifier (f), and ToxVec misclassified instances are compared. In Figures (a) and (f), the red points are venom sequences and the blue points indicate the non-venom sequences. In Figures (b) to (e), the red points indicate the venom sequences identified as non-venom by the predictor and the blue points are the non-venom sequences identified as venom sequences. 
4. The ensemble classifier of different resamples As discussed in step 2, we create $N=10$ training sets resulting in 10 predictive models. We consider a positive sample for the eventual classification output if and only if all 10 models confirm this.

\section{RESULTS}

Venom Protein classification results over the Toxify test set for ToxVec, Toxify, ToxClassifier, and ClanTox are provided in Table 1. For the evaluation, the accuracy, the F1 score of positive and negative classes, and their average (macro-F1) are reported. Our ToxVec outperformed ClanTox, ToxClassifier, and Toxify in terms of $F 1$ on both positive and negative class by improving macro- $F 1$ (average of F1 on positive and negative classes) for 3 percent from 0.86 to 0.89 . Furthermore, the incorporation of negative-set resamplings increased the performance to a macro- $F 1$ of 0.93 .

Table 1. The summary of evaluation results for detecting venom proteins in the Toxify test set: We compare the performance of where ToxVec and its ensemble version with Clantox, ToxClassifier, and Toxify approaches in terms of accuracy, $F 1 \mathrm{~s}$ of both positive and negative classes, and the macro-average of $F 1$ s. The performance of ToxVec for both initialization modes (random initialization and ProtVec-based initialization) are provided.

\begin{tabular}{|l|c|c|c|c|}
\hline \multicolumn{1}{|c|}{ Method } & Accuracy & F1-positive & F1-negative & macro-F1 \\
\hline ClanTox & 0.79 & 0.84 & 0.69 & 0.77 \\
\hline ToxClassifier & 0.73 & 0.78 & 0.65 & 0.72 \\
\hline Toxify & 0.86 & 0.85 & 0.87 & 0.86 \\
\hline \hline ToxVec(Random-init -Emb) & 0.9 & 82 & 0.93 & 0.88 \\
\hline ToxVec-Ensembled $($ Random - init -Emb) & 0.94 & 0.87 & 0.96 & 0.92 \\
\hline \hline ToxVec $($ UniRef $90-$ Emb $)$ & 0.91 & 0.84 & 0.94 & 0.89 \\
\hline ToxVec-Ensembled $($ UniRef $90-$ Emb $)$ & $\mathbf{0 . 9 5}$ & $\mathbf{0 . 8 9}$ & $\mathbf{0 . 9 6}$ & $\mathbf{0 . 9 3}$ \\
\hline
\end{tabular}

We created a t-SNE (Maaten and Hinton, 2008) visualization of the Skip-gram embedding space of protein trimers (Figure 2). In this figure, the trimers of vector size 3000 are mapped into a 2D space. Next, to see how biophysical properties are distributed in this embedding space we color the k-mers for different properties, including mean molecular weight, mean surface accessibility (Emini et al., 1985), mean KD hydrophilicity (Kyte and Doolittle, 1982), mean flexibility (Vihinen et al., 1994), and mean Janin Interior to surface transfer energy scale (Janin et al., 1988). The mentioned biophysical scales are standardized (zero mean and unit variance) to be comparable. Higher intensity (lighter color) indicates being higher in the scales. We can see that the k-mers of similar properties are close in the embedding space. Afterward, we represent Toxify's training instances with the average of their overlapping timers and then mapped them to the 2D space using the same t-SNE projection of simple trimers. The buttom-right sub-figure in Figure 2 shows the venom sequences in red and the non-venoms in blue. Comparison of training instances and the biophysical properties shows the average properties of typical venom sequences versus non-venom protein sequences. The illustration shows that the venoms are diverse in terms of averaged biophysical properties, which is confirmed previously even within certain snake families (Nawarak et al., 2003).

\section{CONCLUSIONS AND DISCUSSIONS}

Here, we described ToxVec, a deep learning model using language model-based representation learning of proteins for venom protein identification. We compared the performance of Tox Vec with recent supervised approaches in venom identification and showed that the supervised fine-tuning of protein language model-based representation achieved state-of-the-art performance in this task. We also addressed the class-imbalance problem in training a predictive model by ensembling models trained on the major class's downsampling, further improving the performance by 4 percent macro F1 (a macro-F1 of 0.93).

Figure 3 showed the visualization of test cases (a), train cases (f, and the misclassified instances using different approaches. The figure suggests that the test cases were not similar to the typical training instances, and the problem has not been trivial for the embedding space. The misclassified instances of 
Toxify, ToxClassifier, and ClanTox follow the same patterns. When Tox Vec was employed, the F1 scores on both venoms/non-venoms classes were improved, which was even better in the negative class.

We observed that the ToxVec outperformed the state-of-the-art venom predictors by $2 \%$ to $7 \%$ macro$\mathrm{F} 1$ (averaged F1 in the positive and negative class). The minimum macro-F1 of ToxVec, 0.88, which was still higher than existing approaches macro-F1 (0.86), was achieved when an embedding layer was trained for k-mers from scratch in a supervised manner. By ensembling ten classifiers trained on different downsampling of the negative set, this performance increased to a macro-F1 of 0.92 . We also showed that when the pretrained Skip-grams over UniRed are used, the macro-F1 and all scores are increased by one more point (macro-F1 $=0.93$ ). These results suggest that automatic feature learning, either by random initialization and then supervised training or fine-tuning of self-supervised embedding, can improve venom identification performance compared to methods using manual feature engineering. Like natural language processing scenarios, fine-tuning of language model-baed representations improved the downstream supervised task performance, which is particularly evident for small training sets. The success of automatic representation learning approaches in our experiments motivates exploring of contextualized embedding (transformers (Rao et al., 2019) or ELMo embeddings (Asgari et al., 2019b; Heinzinger et al., 2019)) as future directions.

\section{REFERENCES}

Asgari, E. (2019). Life Language Processing: Deep Learning-based Language-agnostic Processing of Proteomics, Genomics/Metagenomics, and Human Languages. PhD thesis, UC Berkeley.

Asgari, E., McHardy, A. C., and Mofrad, M. R. (2019a). Probabilistic variable-length segmentation of protein sequences for discriminative motif discovery (dimotif) and sequence embedding (protvecx). Scientific reports, 9(1):1-16.

Asgari, E. and Mofrad, M. R. (2015). Continuous distributed representation of biological sequences for deep proteomics and genomics. PloS One, 10(11):e0141287.

Asgari, E., Poerner, N., McHardy, A., and Mofrad, M. (2019b). Deepprime2sec: Deep learning for protein secondary structure prediction from the primary sequences. bioRxiv, page 705426 .

Atchley, W. R., Zhao, J., Fernandes, A. D., and Drüke, T. (2005). Solving the protein sequence metric problem. Proceedings of the National Academy of Sciences, 102(18):6395-6400.

Bengio, Y. (2012). Deep learning of representations for unsupervised and transfer learning. In Proceedings of ICML workshop on unsupervised and transfer learning, pages 17-36.

Bojanowski, P., Grave, E., Joulin, A., and Mikolov, T. (2017). Enriching word vectors with subword information. Transactions of the Association for Computational Linguistics, 5:135-146.

Casewell, N. R., Wüster, W., Vonk, F. J., Harrison, R. A., and Fry, B. G. (2013). Complex cocktails: the evolutionary novelty of venoms. Trends in ecology \& evolution, 28(4):219-229.

Cho, K., van Merriënboer, B., Gulcehre, C., Bahdanau, D., Bougares, F., Schwenk, H., and Bengio, Y. (2014). Learning phrase representations using RNN encoder-decoder for statistical machine translation. In Proceedings of the 2014 Conference on Empirical Methods in Natural Language Processing $(E M N L P)$, pages 1724-1734, Doha, Qatar. Association for Computational Linguistics.

Cole, T. J. and Brewer, M. S. (2019). Toxify: a deep learning approach to classify animal venom proteins. PeerJ, 7:e7200.

Cortes, C. and Vapnik, V. (1995). Support-vector networks. Machine learning, 20(3):273-297.

Dao, F.-Y., Yang, H., Su, Z.-D., Yang, W., Wu, Y., Hui, D., Chen, W., Tang, H., and Lin, H. (2017). Recent advances in conotoxin classification by using machine learning methods. Molecules, 22(7):1057.

Emini, E. A., Hughes, J. V., Perlow, D., and Boger, J. (1985). Induction of hepatitis a virus-neutralizing antibody by a virus-specific synthetic peptide. J. Virology, 55(3):836-839.

Friedman, J. H. (2002). Stochastic gradient boosting. Computational statistics \& data analysis, 38(4):367378.

Gacesa, R., Barlow, D. J., and Long, P. F. (2016). Machine learning can differentiate venom toxins from other proteins having non-toxic physiological functions. PeerJ Computer Science, 2:e90.

Goldberg, Y. and Levy, O. (2014). word2vec explained: deriving mikolov et al.'s negative-sampling word-embedding method. arXiv preprint arXiv:1402.3722.

Hargreaves, A. D., Swain, M. T., Hegarty, M. J., Logan, D. W., and Mulley, J. F. (2014). Restriction and recruitment - gene duplication and the origin and evolution of snake venom toxins. Genome biology and evolution, 6(8):2088-2095. 
Heinzinger, M., Elnaggar, A., Wang, Y., Dallago, C., Nechaev, D., Matthes, F., and Rost, B. (2019). Modeling aspects of the language of life through transfer-learning protein sequences. BMC bioinformatics, 20(1):723.

Howard, J. and Ruder, S. (2018). Universal language model fine-tuning for text classification. In Proceedings of the 56th Annual Meeting of the Association for Computational Linguistics (Volume 1: Long Papers), pages 328-339, Melbourne, Australia. Association for Computational Linguistics.

Iba, W. and Langley, P. (1992). Induction of one-level decision trees. In Machine Learning Proceedings 1992, pages 233-240. Elsevier.

Janin, J., Miller, S., and Chothia, C. (1988). Surface, subunit interfaces and interior of oligomeric proteins. Journal of molecular biology, 204(1):155-164.

Jenner, R. A., von Reumont, B. M., Campbell, L. I., and Undheim, E. A. B. (2019). Parallel Evolution of Complex Centipede Venoms Revealed by Comparative Proteotranscriptomic Analyses. Molecular Biology and Evolution, 36(12):2748-2763.

Jungo, F., Bougueleret, L., Xenarios, I., and Poux, S. (2012). The uniprotkb/swiss-prot tox-prot program: a central hub of integrated venom protein data. Toxicon, 60(4):551-557.

Kyte, J. and Doolittle, R. F. (1982). A simple method for displaying the hydropathic character of a protein. J. Mol. Biol., 157(1):105-132.

Lewis, R. J. and Garcia, M. L. (2003). Therapeutic potential of venom peptides. Nature reviews drug discovery, 2(10):790-802.

Linial, M., Rappoport, N., and Ofer, D. (2017). Overlooked short toxin-like proteins: a shortcut to drug design. Toxins, 9(11):350.

Maaten, L. v. d. and Hinton, G. (2008). Visualizing data using t-sne. Journal of machine learning research, 9(Nov):2579-2605.

Mikolov, T., Sutskever, I., Chen, K., Corrado, G. S., and Dean, J. (2013). Distributed representations of words and phrases and their compositionality. In Advances in neural information processing systems, pages 3111-3119.

Naamati, G., Askenazi, M., and Linial, M. (2009). Clantox: a classifier of short animal toxins. Nucleic acids research, 37(suppl_2):W363-W368.

Nawarak, J., Sinchaikul, S., Wu, C.-Y., Liau, M.-Y., Phutrakul, S., and Chen, S.-T. (2003). Proteomics of snake venoms from elapidae and viperidae families by multidimensional chromatographic methods. Electrophoresis, 24(16):2838-2854.

Nelder, J. A. and Wedderburn, R. W. (1972). Generalized linear models. Journal of the Royal Statistical Society: Series A (General), 135(3):370-384.

Ojeda, P. G., Ramírez, D., Alzate-Morales, J., Caballero, J., Kaas, Q., and González, W. (2018). Computational studies of snake venom toxins. Toxins, 10(1):8.

Pan, X., Zuallaert, J., Wang, X., Shen, H.-B., Campos, E. P., Marushchak, D. O., and De Neve, W. (2020). Toxdl: Deep learning using primary structure and domain embeddings for assessing protein toxicity. Bioinformatics.

Prashanth, J. R., Hasaballah, N., and Vetter, I. (2017). Pharmacological screening technologies for venom peptide discovery. Neuropharmacology, 127:4-19.

Rao, R., Bhattacharya, N., Thomas, N., Duan, Y., Chen, P., Canny, J., Abbeel, P., and Song, Y. (2019). Evaluating protein transfer learning with tape. In Advances in Neural Information Processing Systems, pages 9689-9701.

Starcevic, A., Moura-da Silva, A. M., Cullum, J., Hranueli, D., and Long, P. F. (2015). Combinations of long peptide sequence blocks can be used to describe toxin diversification in venomous animals. Toxicon, 95:84-92.

Tan, C., Sun, F., Kong, T., Zhang, W., Yang, C., and Liu, C. (2018). A survey on deep transfer learning. In International conference on artificial neural networks, pages 270-279. Springer.

Vihinen, M., Torkkila, E., and Riikonen, P. (1994). Accuracy of protein flexibility predictions. Proteins, 19(2):141-149.

Wan, F. and Zeng, J. M. (2016). Deep learning with feature embedding for compound-protein interaction prediction. bioRxiv, page 086033 .

Wolf, T., Debut, L., Sanh, V., Chaumond, J., Delangue, C., Moi, A., Cistac, P., Rault, T., Louf, R., Funtowicz, M., et al. (2019). Huggingface's transformers: State-of-the-art natural language processing. ArXiv, pages arXiv-1910. 
bioRxiv preprint doi: https://doi.org/10.1101/2020.09.29.319046; this version posted October 1, 2020. The copyright holder for this preprint (which was not certified by peer review) is the author/funder. All rights reserved. No reuse allowed without permission.

${ }_{303}$ Wong, E. S., Hardy, M. C., Wood, D., Bailey, T., and King, G. F. (2013). Svm-based prediction of 304 propeptide cleavage sites in spider toxins identifies toxin innovation in an australian tarantula. PLoS 305 One, 8(7):e66279. 